Running Head: DOES THE MEDIUM MATTER?: A COMPARISON OF

Does the Medium MatTer?: A COMPARISON OF A Web-BASED TUtORIAL With

FACE-TO-FACE LIBRARY INSTRUCTION ON EDUCATION STUDENTS’

SELF-EFFICACY LEVELS AND LEARNING OUTCOMES

Penny M. Beile and David N. Boote

University of Central Florida

Author Info:

Penny M. Beile, Associate Librarian David N. Boote, Assistant Professor

University of Central Florida University of Central Florida

UCF Libraries Department of Educational Studies

pbeile@mail.ucf.edu $\quad$ dboote@mail.ucf.edu 


\begin{abstract}
The purpose of this study was to explore if students’ library skills self-efficacy levels and learning outcomes varied based on instructional delivery mode. Groups consisted of an on-campus class with face-to-face instruction, an on-campus class with a Web-based library tutorial, and a Web-based class with a Web-based tutorial. Data were collected immediately prior to instruction and again six weeks after. Analysis indicated self-efficacy levels and learning outcomes significantly increased across all groups after instruction. Groups varied significantly on final self-efficacy levels, but not on final library skills scores. Results are discussed as they relate to the viability of Web-based tutorials for library instruction.
\end{abstract}


Does the medium matter?: A comparison of a Web-based tutorial with face-to-face library instruction on education students' self-efficacy levels and learning outcomes Among the first entities to recognize the potential of technology to facilitate information delivery, academic libraries quickly capitalized on this phenomenon by creating electronic card catalogs and providing desktop access to library databases. However, with the explosion in the number of distance classes, programs, and degrees being developed and offered in higher education, libraries are being challenged to provide quality library instruction to the off-campus user. Many libraries are creating Web-based library tutorials to accommodate the off-campus student, but little research has been reported investigating the effect of Web-based library tutorials as compared to face-to-face library instruction on student self-efficacy levels and learning outcomes.

Perceived self-efficacy is a well-established construct that suggests people are more likely to engage in activities in which they feel efficacious. A component of Albert Bandura's (1977) social cognitive learning theory, self-efficacy is generally defined as the belief in one's ability to successfully perform a given behavior. Partly on the basis of self-percepts of efficacy, people choose what to do, the amount of effort to invest in activities, and how long to persevere at them. Whether a person will make an effort to handle a given situation depends on the strength of his or her effectiveness beliefs.

Bandura (1997) went on to suggest that the skills and characteristics constituting the construct of self-efficacy are alterable by interventions. That is, students may learn to develop and increase affective factors that are associated with performance. Thus, selfefficacy research is meaningful as it is hypothesized that increased time spent at task may translate into better performance and, in turn, positively impact performance outcome. 
However, only a limited number of studies have examined students’ psychosocial perceptions of their library skills, or explored the relationship between the affective domain and cognitive gains after exposure to library instruction (Greer,Weston, \& Alm, 1991; Maughan, 2001; Nahl-Jakobovits \& Jakobovits, 1993; Ren, 2000). Further, none of these studies extended to comparisons between different learning environments, or compared face-to-face with Web-based delivery of instruction. With the early foray of libraries into the electronic medium, it is surprising that no library research has been reported that compares self-efficacy levels and learning outcomes across different learning environments.

\section{Review of Literature}

Identifying, accessing, searching, retrieving, and evaluating information is a complex process involving both the affective and cognitive domains. Thus, this investigation is informed by the studies of Ren (2000), Fox and Weston (1993), Maughan (2001), and Greer, Weston, and Alm (1991), who comprise the bulk of research comparing library skills self-efficacy and learning outcomes. Wen-Hua Ren (2000) examined information searching self-efficacy as it related to electronic library databases by surveying 85 students prior to and after participation in a face-to-face library instruction session. Ren reported participants' scores on a measure of self-efficacy significantly increased between pre-instruction and post-instruction. Post-instruction selfefficacy scores were also significantly correlated with grade on a library assignment; as self-efficacy scores increased, so did grades.

In a similar study, Fox and Weston (1993) compared students receiving courseintegrated library instruction in a nursing program with students who did not receive 
formal instruction. In addition to taking a test to measure actual skills, library users selfassessed their perceived ability to successfully use library resources. Based on analysis of survey data, researchers reported students who participated in course-integrated library instruction had higher self-awareness and self-confidence levels than those who did not participate. However, these results did not necessarily translate into higher levels of general library literacy when compared to actual gains in skills.

Other research has also failed to find a significant relationship between selfefficacy scores and actual learning gains. An instructional assessment study of graduating seniors from various departments undertaken by the University of California - Berkeley revealed those surveyed held a higher opinion of their library research skills than they were able to demonstrate by their test scores (Maughan, 2001). Greer, Weston, and Alm (1991) also reported that while self-assessed library skills were markedly higher for seniors than for freshmen, there was no dramatic trend of increased proficiency from freshmen to seniors in scores on a measure of library skills.

Although some researchers suggest integrating the affective domain with library skills learning in library instruction (Martin, 1989; Nahl-Jakobovits \& Jakobovits, 1993; Ren, 2000), little is still known about the impact of library instruction on self-efficacy and learning outcomes, or the correlation between self-efficacy and the demonstrated ability to perform library research. Further, of the studies that analyzed self-efficacy levels and actual library skills both before and after library instruction, none compared different instructional modes. Web-delivered instruction adds another dimension to the traditional model, and the current study extends research in this area by comparing student performance across different instructional settings. 
The purpose of the present study was to examine the relative impact of three instructional settings: an on-campus class attending a face-to-face library instruction session, an on-campus class completing a Web-based library tutorial, and a Web-based class completing a Web-based library tutorial, on self-efficacy levels and learning of library research skills among education graduate students. Three hypotheses for the study were:

1) Higher levels of library skills self-efficacy would be positively correlated to higher scores on a library skills quiz.

2) Library skills self-efficacy levels would increase after instruction, but would not significantly vary across learning environment.

3) Library skills quiz scores would increase after instruction, but would not significantly vary across learning environment.

Method

\section{Participants and Design}

Participants in the study were 49 masters, doctoral, and certificate-seeking Education students (40 females, 9 males) enrolled in one of three sections of a graduatelevel research methods course at a large, urban university. The research methods course was selected for the study as multiple sections are offered each semester and at least one is wholly Web-based. Also, as students are expected to perform a comprehensive review of the literature in partial fulfillment of the course requirement, course professors traditionally request a library instruction session prior to the review of literature assignment. Students within the classes were selected as study participants based on the 
criteria of enrollment in the course, anticipation of receiving formal library instruction, and agreement to participate in the study.

Library instruction, as the independent variable, consisted of three conditions: Group 1, an on-campus class with face-to-face library instruction; Group 2, an on-campus class with Web-based library tutorial; and Group 3, a Web-based class with a Web-based library tutorial. Comparisons of Web-based and face-to-face instruction generally rely solely on students who have self-selected that particular instructional setting. For example, only students who have chosen to enroll in a campus-based class receive faceto-face instruction. Likewise, students enrolled in Web-based classes receive Web-based instruction. For this particular design, researchers were interested in the results of a class that did not self-select for a particular mode of instruction; as such, Group 2 is the class of particular interest in this study. There were two within-subjects factors, library skills self-efficacy levels and knowledge of library skills as indicated by quiz score.

A repeated measures multivariate analysis of variance (MANOVA) was performed with scores on a library skills self-efficacy survey and a library skills quiz as repeated (pretreatment and posttreatment) dependent variables. The MANOVA test is traditionally deemed appropriate for this analysis as it controls for correlations between dependent variables, thus providing a single test for multiple dependent variables and reducing the possibility of Type 1 error. However, Dugard and Todman (1995) suggest in their analysis of repeated measures designs in educational research that using pretreatment scores as covariates may provide a more appropriate and informative analysis. As such, a multivariate analysis of covariance (MANCOVA), using prior 
library instruction (indicated by exploratory analysis) and pretreatment scores for library skills self-efficacy levels and library skills quiz scores as covariates, was also performed.

\section{Instrumentation}

Self-efficacy scores were determined by responses on a library skills self-efficacy scale, which was grounded on self-percepts of ability to successfully use educationspecific information sources. The instrument consisted of 30 items designed to measure students’ perceptions of their ability to successfully perform library research. Participants responded to statements such as "I can identify equivalent or related search terms," "I can search for books by author in the library catalog," and "I can easily differentiate between primary and secondary resources” by indicating how strongly they agreed with the statement on a five-point Likert-type scale, ranging from 1 (strongly disagree) to 5 (strongly agree).

Library skills levels were indicated by performance on a test of library skills written by faculty who teach library instruction sessions and based on ACRL information literacy objectives (Association of College and Research Libraries, 2000). The library skills quiz, which consisted of 20 multiple-choice questions, was designed to assess conceptual knowledge (how information is produced and organized), knowledge of database searching skills (identifying databases and using Boolean logic), and knowledge of institution-specific information (accessing databases and awareness of services).

\section{Procedures}

All students enrolled in one of the selected research methods course sections were invited to participate. Participants in the face-to-face classroom setting were orally informed of the study and given instructions on how to complete the survey. Written 
instructions were offered via course email for students enrolled in the Web-based class.

The pretreatment measures were administered to assess students' self-perceived ability to successfully perform library research and knowledge of library skills prior to instruction. Surveys were distributed and completed immediately before the instructional session. Combined (self-efficacy measure and library skills quiz), the survey took approximately 15 minutes to complete. Fifty-eight surveys were returned; Group 1 (on-campus class /face-to-face instruction) returned 16 surveys, Group 2 (on-campus class/Web-based tutorial) returned 28, and Group 3 (Web-based class/Web-based tutorial) returned 14.

Testing was repeated approximately six weeks after the instructional session. Forty-nine posttreatment surveys were returned, thus rendering nine pretreatment measures unusable. Return rates for Group 1 were 16 (100\%), Group 2 were 19 (68\%), and Group 3 were 14 (100\%). Between instruction session and final administration of the instruments, participants were expected to complete a review of the literature as a class assignment.

As the treatment with three conditions, library instruction sessions were administered to all participating students. Face-to-face instruction for the on-campus class consisted of a 70-minute demonstration of relevant library databases, followed by an activity to allow the students to immediately apply the lesson. The Web-based library tutorial $^{1}$ consisted of four interactive modules, on which participants spent an average of 80 minutes. Students in different sections of the research methods class, one on-campus class and one Web-based class, completed the tutorial.

\footnotetext{
${ }^{1}$ The Web-based tutorial used in this study was developed exclusively for education information sources. The four interactive modules include: principles of library and information research, navigation and search techniques, practical application of search techniques, and locating, evaluating, and citing information. The tutorial is located at http://library.ucf.edu/cmc/edtut.
} 
Results

\section{Preliminary Analysis}

Pretreatment self-efficacy scores ranged from 18 to 114 out of a maximum of 150 . Final self-efficacy scores ranged from 43 to 118. The average self-efficacy level of participants across all classes increased from a mean of $68.88(\mathrm{SD}=19.92)$ before instruction session to a mean of $91.90(\mathrm{SD}=16.24)$ after. Table 1 presents descriptive statistics for self-efficacy levels by group; see Figure 1 for a graphical display of scores.

Pretreatment library skills quiz scores ranged from 30 to 95 out of a maximum of 100. Final library skills quiz scores ranged from 50 to 95 . The average library skills quiz score of participants across all classes increased from a mean of $58.78(\mathrm{SD}=13.86)$ before instruction to a mean of $73.16(\mathrm{SD}=12.65)$ after. Table 2 contains descriptive statistics for library skills scores by group; Figure 2 graphically displays mean scores.

An independent $t$-test was also calculated comparing mean scores of participants who had received prior library instruction $(n=18)$ with mean scores of participants who had not attended a prior library instruction session $(n=31)$. A significant difference between the means of the two groups was indicated on three variables; participants with prior library instruction differed from those without on pretreatment self-efficacy levels $(t(47)=2.37, \mathrm{p}<.05)$, final self-efficacy levels $(t(47)=2.13, \mathrm{p}<.05)$, and final library skills scores $(t(47)=2.00, \mathrm{p}<.05)$. No statistically significant difference was found when comparing mean scores between the two groups on pretreatment library skills quiz scores $(t(47)=1.23, \mathrm{p}=.26)$. Students who had been previously exposed to library instruction reported higher self-efficacy levels and scored higher on the final library skills 
quiz. Table 3 presents descriptive statistics for prior library instruction exposure by pretreatment and final self-efficacy levels and library skills quiz scores. Main analysis

Pearson correlation coefficients were calculated between both pretreatment selfefficacy levels library skills quiz scores and final scores. Each indicated a moderate positive statistically significant correlation (pretreatment $r=.39, \mathrm{p}<.05$ and posttreatment $r=.39, \mathrm{p}<.05$ ) that accounted for $16 \%$ of the variance in scores. Thus, hypothesis 1 received support in that as library skills self-efficacy levels increased, so did library skills quiz scores.

Multivariate analysis was deemed appropriate as statistically significant correlations between the dependent variables met the assumptions of relationship. A repeated measures multivariate analysis of variance (MANOVA) was calculated comparing library skills self-efficacy levels and library skills quiz scores immediately prior to instruction and again approximately six weeks later. A statistically significant effect was found for self-efficacy levels $(F(1,48)=47.72$, $\mathrm{p}<.05)$. Follow-up dependent $t$-tests revealed self-efficacy levels increased significantly $(t(48)=-9.28, \mathrm{P}<.01)$ from before instruction $(M=68.88, S D=19.92)$ to after $(M=91.90, S D=16.24)$, with instruction accounting for $50 \%$ of the variance in scores.

A statistically significant effect was also found for library skills quiz scores $(\mathrm{F}(1,48)=124.11, \mathrm{p}<.05)$. Follow-up dependent $t$-tests indicated library skills quiz scores increased significantly $(t(48)=-7.51, \mathrm{p}<.05)$ from before instruction $(\mathrm{M}=58.78$, $\mathrm{SD}=13.86)$ to after $(\mathrm{M}=73.16, \mathrm{SD}=12.65)$, with instruction accounting for $72 \%$ of the variance. Both self-efficacy levels and library skills quiz scores significantly increased 
after instruction, and variance measures indicate instruction accounted for the majority of the increase. The first parts of hypothesis 2 and hypothesis 3, which posited that library skills self-efficacy levels and quiz scores would increase after instruction, was supported.

Covariate analysis also requires that the covariate be statistically significantly related to the dependent variable. A Pearson correlation coefficient calculation indicated pretreatment and final self-efficacy levels were statistically significantly related $(r=.56$, $\mathrm{p}<.05$ ), as were pretreatment library skills quiz scores and final library skills quiz scores $(r=.49, \mathrm{p}<.05)$. Prior library instruction was also significantly correlated with final self-efficacy levels $(r=.30, \mathrm{p}<.05)$ and final library skills quiz scores $(r=.28, \mathrm{p}<.05)$, thus meeting the relationship assumptions. As such, a multivariate analysis of covariance (MANCOVA), with pre-instruction self-efficacy levels and library skills quiz scores as covariates, was deemed the appropriate statistical procedure. Due to its significant effect on final self-efficacy levels and library skills quiz scores, exposure to prior library instruction was also analyzed as a covariate.

The MANCOVA procedure, with library instruction as the independent variable and pretreatment self-efficacy levels, pretreatment library skills quiz scores, and prior library instruction as covariates indicated a statistically significant difference among the three treatment conditions $(\mathrm{F}(4,84)=2.52, \mathrm{p}<.05)$. Univariate $F s$, in between-subjects effects, revealed significant group differences on final self-efficacy levels $(F(2,43)=$ 3.97, $\mathrm{p}<.05$ ). Follow-up analyses revealed that Group 3, Web-based class/Web tutorial (adj $\mathrm{M}=98.70, \mathrm{SD}=11.77$ ) demonstrated significantly higher self-efficacy levels than Group 2, on-campus students/Web tutorial (adj $\mathrm{M}=85.62, \mathrm{SD}=18.09),(\mathrm{F}(2,46)=6.59$, $\mathrm{p}<.05$ ). This finding did not support one expectation of hypothesis 2 , which stated that 
library skills self-efficacy levels would not vary across learning environment. No statistically significant differences were found across classes on final library skills quiz scores $(\mathrm{F}(2,43)=1.40, \mathrm{p}=.26)$, thereby supporting the second part of hypothesis 3 , that library skills quiz scores would not vary across learning environment. All groups demonstrated similar gains in library skills quiz scores across learning environments.

\section{Limitations of the Study}

The viability of utilizing Web-based tutorials for library instruction, at least for graduate students in education, is partially substantiated by this study. However, as only performance on a measure of library skills and attitudinal responses on a survey were analyzed, additional research should be conducted using other performance measures. Empirical observation of actual performance and analyzing the quality of references in a research bibliography are two methods that might yield more authentic outcomes.

Additionally, the fact that the library skills self-efficacy survey and library skills quiz have yet to be validated with this sample raises concern. Exploratory analysis of the library skills self-efficacy survey has been performed with a prior sample $(n=42)$ [Beile, 2001], but further analysis with this sample is warranted. Exploratory analysis revealed a four-factor solution that explained $56.2 \%$ of the systematic covariance among the items and offered a reliability alpha of .85 .

Finally, the possibility that the relatively small sample is not representative of the overall population of education graduate students must be acknowledged. Individuals within the course sections volunteered for the study, thus creating inherent bias problems as volunteers may be more likely to perceive positively their ability to successfully 
perform library research. Results of this study would be more tenable by replication with a larger, randomly selected sample.

\section{Discussion}

The purpose of the study was to examine the relative impact of three different library instruction learning environments on self-efficacy levels and learning outcomes for graduate students in education. In this study, the treatment of library instruction, regardless of condition, resulted in significantly greater levels of self-efficacy. Also, students who felt more efficacious demonstrated higher scores on the library skills quiz. These findings are consistent with Martin (1989), Nahl-Jakobovits and Jakobovits (1993) and Ren (2000), who have suggested that within the context of library skills, increased levels of self-efficacy are positively related to greater learning outcomes. Whether developing Web-based tutorials or delivering face-to-face instruction, these findings suggest librarians would do well to attend to the affective domain as well as the cognitive.

Again, regardless of instructional environment or condition, all groups significantly improved their library skills scores. This finding offers contradictory evidence to Eadie’s (1990) assertion that library instruction provides little in the way of results and is of marginal importance for educating the library user. Further, repeated exposure to library instruction appears to offer a positive effect on both self-efficacy levels and library skills quiz scores. This pattern suggests that repeated library instruction may have a cumulative effect on learning. If further investigation clarifies this relationship, then support would be provided that students could benefit from repeated library instruction throughout their academic careers. 
Between-group patterns were not as easy to discern. Although differences in library skills quiz scores were not statistically significant between groups, statistical significance was found in gains in self-efficacy levels. Final self-efficacy levels between Group 2 (on-campus class/Web-based tutorial) and Group 3 (Web-based class/Webbased tutorial) were statistically significantly different. Group 2 demonstrated the greatest learning gains, but reported the lowest gains in self-efficacy in between-group comparisons. Two possible explanations come quickly to mind. First, it is possible that Group 2, the on-campus class students, were less familiar than Group 3 with the Webbased tutorial and therefore attended to the instruction more. However, it is equally plausible to consider that the self-efficacy levels of Group 3 were related to their experience and facility with Web-based instruction, and thus produced a positive range effect for the group.

Perhaps most importantly, it is possible that mixing instructional modes which students do not self-select for may reveal the greatest understanding of research on the learner/mode dynamic. This mixed instructional mode research design has been relatively unreported in the literature, but may offer the most complete understanding of the learning environment effect on users.

\section{Conclusions}

These results suggest at least three practical recommendations. First, as the growth of Web-based instruction continues, libraries and colleges will continue to be challenged to deliver instruction that meets user demand for mediating off-campus access to information resources. At least in this instance, library instruction delivered via a Web-based tutorial supported students as effectively as face-to-face instruction, thus 
appearing to meet the need for off-campus instruction to information resources. Students in the Web-based class further indicated they felt the tutorial was useful in assisting with their review of the literature assignment, and one student noted that she used the tutorial as a reference, consulting it whenever she had a question or needed to refresh her knowledge of database searching.

Second, a Web-based library tutorial such as the one used in this study may produce the same cognitive outcomes as face-to-face library instruction. As learning gains were similar for both on-campus and Web-based classes completing the tutorial, a Web-based tutorial may be a viable replacement for traditional library instruction sessions. As course syllabi become increasingly more compressed a Web-based tutorial may provide some relief by replacing class time devoted to library instruction. Some of the fears of replacing face-to-face interaction with a tutorial seem unwarranted in terms of impact on academic performance. These results may offer instructors a choice of strategies that can be employed with students throughout their programs.

Finally, regardless of learning environment, library instruction appears to positively influence both library skills self-efficacy levels and learning outcomes. Further, repeated exposure to library instruction may have a cumulative effect on student learning and self-efficacy. Effective library skills enable students to search, retrieve, and critically evaluate information for their personal and academic needs, and instruction for library skills should be an integral part of program curricula. 


\section{References}

Association of College and Research Libraries, Task Force on Information Literacy Competency Standards. (2000). Information Literacy Competency Standards for Higher Education. Retrieved July 11, 2003, from http://www.ala.org/Content/NavigationMenu/ACRL/Standards_and_Guidelines/I nformation_Literacy_Competency_Standards_for_Higher_Education.htm

Bandura, A. J. (1977). Self-efficacy: Toward a unifying theory of behavioral change. Psychological Review, 84(2), 191-215.

Bandura, A. J. (1997). Self-efficacy: The exercise of control. New York: Freeman.

Beile, P. M. (2001). [Factor analysis of library skills self-efficacy survey]. Unpublished raw data.

Dugard, P. \& Todman, J. (1995). Analysis of pre-test-post-test control group designs in educational research. Educational Psychology, 15(2), 181-199.

Eadie, T. (1990). Immodest proposals. Library Journal, 115(7), 42-45.

Fox, L. M. \& Weston, L. (1993). Course-integrated instruction for nursing students: How effective?. Research Strategies, 11(2), 89-99.

Greer, A., Weston, L., \& Alm, M. L. (1991). Assessment of learning outcomes: A measure of progress in library literacy. College \& Research Libraries, 52, 549557.

Martin, B. L. (1989). A checklist for designing instruction in the affective domain. Educational Technology, 29(8), 7-15.

Maughan, P. D., (2001). Assessing information literacy among undergraduates: A 
discussion of the literature and the University of California-Berkeley experience. College \& Research Libraries, 62(1), 71-85.

Nahl-Jakobovits, D. \& Jakobovits, L. A. (1993). Bibliographic instructional design for information literacy: Integrating affective and cognitive objectives. Research Strategies, 11, 73-88.

Ren, W. H. (2000). Library instruction and college student self-efficacy in electronic information searching. Journal of Academic Librarianship, 26(5), 323-328. 


\section{TABLE 1}

Descriptive statistics for self-efficacy levels, by Group

\begin{tabular}{cccc}
\hline & Mean & SD & $n$ \\
\hline Pretreatment Self-efficacy Levels & & & \\
Group 1 & 65.06 & 16.25 & 16 \\
Group 2 & 64.74 & 21.89 & 19 \\
Group 3 & 78.86 & 18.63 & 14 \\
Total & 68.88 & 19.92 & 49 \\
Final Self-efficacy Levels & & & 16 \\
Group 1 & 92.50 & 11.96 & 19 \\
Group 2 & 83.68 & 18.09 & 49 \\
Group 3 & 102.36 & 11.77 & 14 \\
Total & 91.90 & 16.24 & \\
& & & \\
\hline
\end{tabular}

Note: $\quad$ Maximum Self-efficacy Score $=150$

Group 1 - on-campus class with face-to-face library instruction session

Group 2 - on-campus class with Web-based library tutorial

Group 3 - Web-based class with Web-based library tutorial 


\section{TABLE 2}

Descriptive statistics for library skills quiz scores, by Group

\begin{tabular}{cccc}
\hline & Mean & SD & $n$ \\
\hline Pretreatment Library Skills Scores & & & \\
Group 1 & 60.00 & 9.83 & 16 \\
Group 2 & 54.21 & 14.65 & 19 \\
Group 3 & 63.57 & 15.62 & 14 \\
Total & 58.78 & 13.86 & 49 \\
Final Library Skills Scores & & & \\
Group 1 & 70.63 & 11.53 & 16 \\
Group 2 & 71.32 & 12.00 & 19 \\
Group 3 & 78.57 & 13.93 & 14 \\
Total & 73.16 & 12.65 & 49 \\
\hline
\end{tabular}

Note: $\quad$ Maximum Library Skills Score $=100$

Group 1 - on-campus class with face-to-face library instruction session

Group 2 - on-campus class with Web-based library tutorial

Group 3 - Web-based class with Web-based library tutorial 
TABLE 3

Descriptive statistics, participants’ scores with and without prior library instruction

\begin{tabular}{|c|c|c|c|c|}
\hline & \multicolumn{2}{|c|}{ With prior LI (n18) } & \multicolumn{2}{|c|}{ Without prior LI (n31) } \\
\hline & Mean & SD & Mean & SD \\
\hline Pretreatment Self-efficacy & 77.33 & 18.45 & 63.97 & 19.35 \\
\hline Pretreatment Library Skills & 61.94 & 12.50 & 56.94 & 14.47 \\
\hline Final Self-efficacy & 98.17 & 14.60 & 88.26 & 16.24 \\
\hline Final Library Skills & 77.78 & 12.27 & 70.48 & 12.27 \\
\hline
\end{tabular}

Note: Maximum Self-efficacy Score $=150$

Maximum Library Skills Score $=100$ 


\section{FIGURE 1}

Pretreatment and final library skills self-efficacy levels, by Group

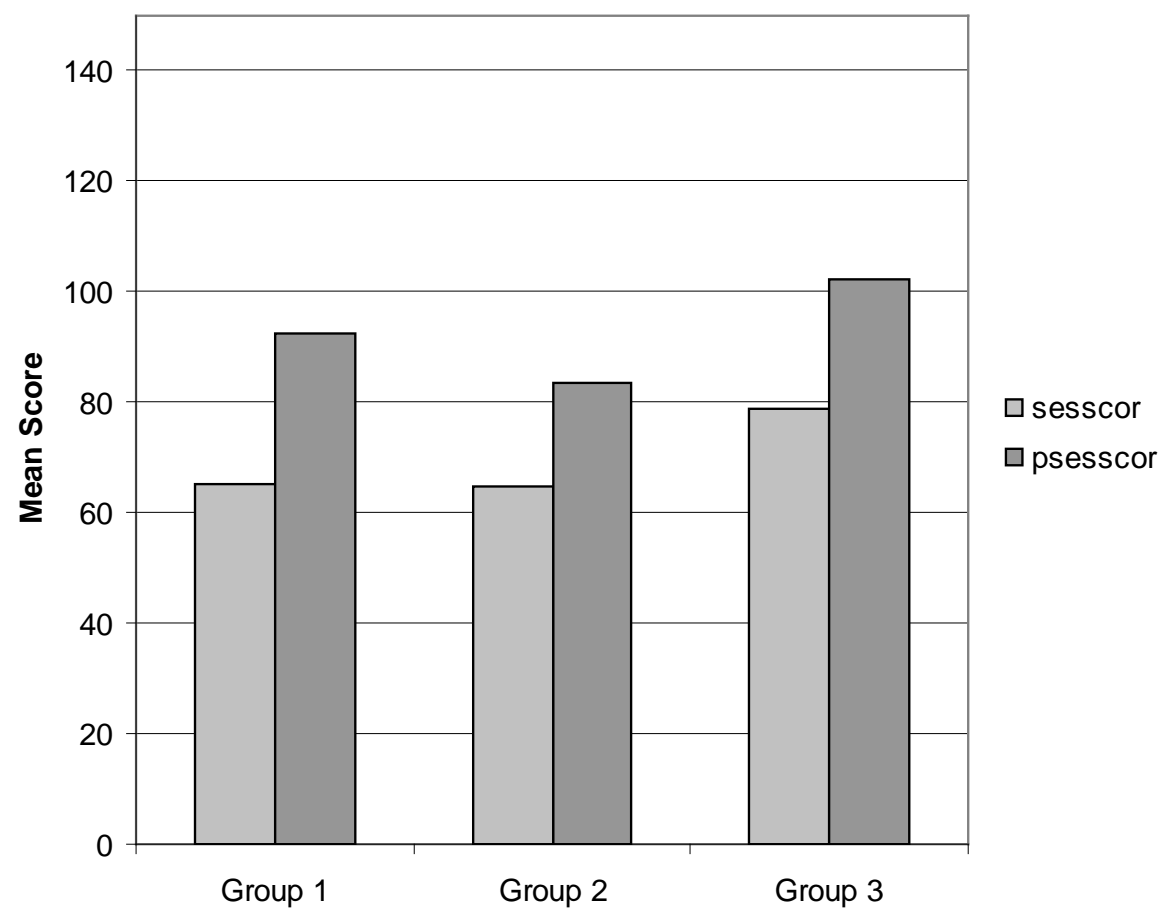

$$
\begin{aligned}
& \text { SESSCOR = pretreatment scores } \\
& \text { PSESSCOR = final scores }
\end{aligned}
$$

Maximum Self-efficacy Score $=150$

Group 1 - on-campus class with face-to-face library instruction session

Group 2 - on-campus class with Web-based library tutorial

Group 3 - Web-based class with Web-based library tutorial 


\section{FIGURE 2}

Pretreatment and final library skills quiz scores, by Group

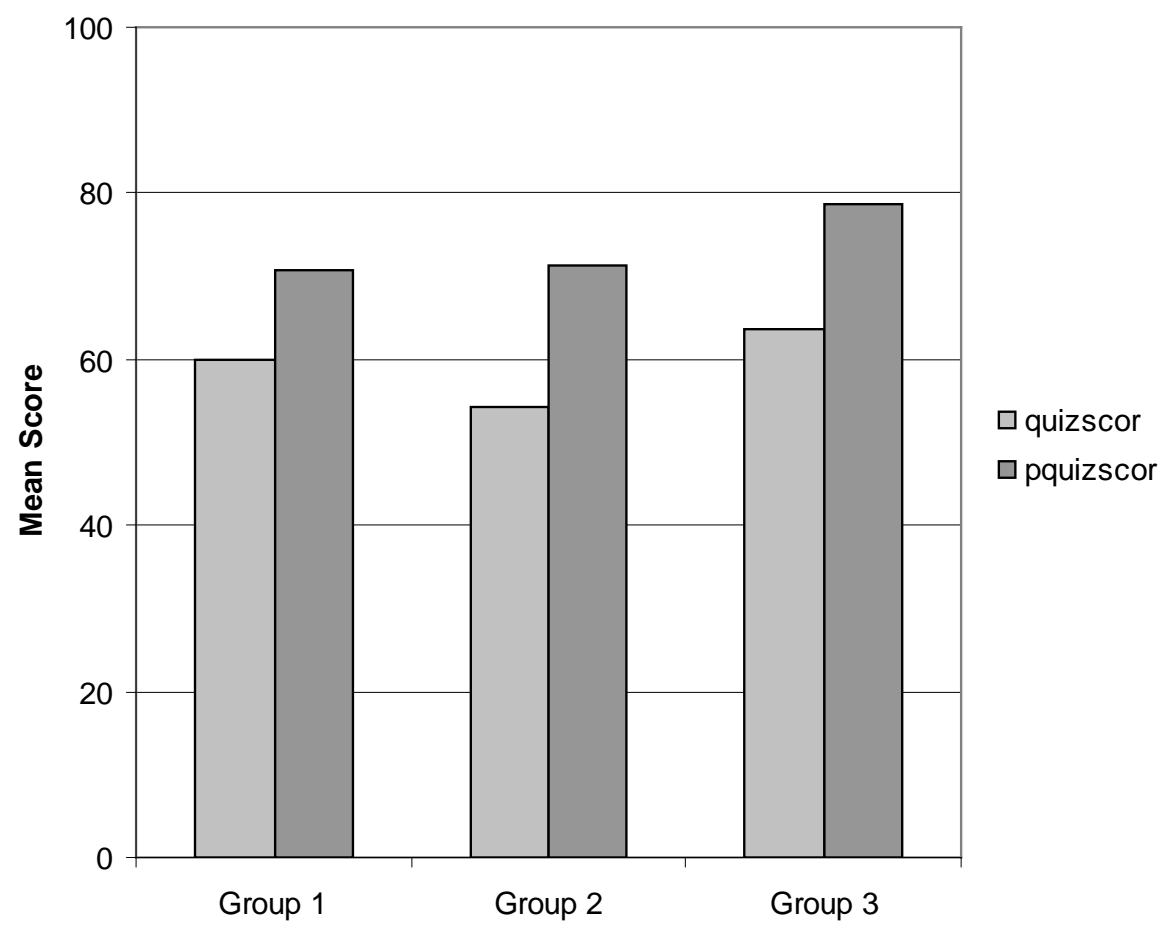

QUIZSCOR = pretreatment scores

PQUIZSCOR = final scores

Maximum Library Skills Score $=100$

Group 1 - on-campus class with face-to-face library instruction session

Group 2 - on-campus class with Web-based library tutorial

Group 3 - Web-based class with Web-based library tutorial 